\title{
Spatiotemporal expression of zebrafish keratin 18 during early embryogenesis and the establishment of a keratin 18:RFP transgenic line
}

\author{
Yun-Hsin Wang ${ }^{\text {a,1, }}$, Yau-Hung Chen ${ }^{\text {b,1 }}{ }^{\text {, Yu-Ju Lin }}{ }^{\text {a }}$, Huai-Jen Tsai ${ }^{\text {a, } *}$ \\ ${ }^{a}$ Institute of Molecular and Cellular Biology, National Taiwan University No. 1, Roosevelt Road, Section 4, Taipei 106, Taiwan, ROC \\ ${ }^{\mathrm{b}}$ Graduate Institute of Life Sciences, Tamkang University, Tamshui, Taiwan, ROC
}

Received 24 August 2005; received in revised form 13 October 2005; accepted 14 October 2005

Available online 30 January 2006

\begin{abstract}
Zebrafish cytokeratin 18 (K18) is one of the type I keratin genes expressed the earliest after amputation of the zebrafish fin, but its spatiotemporal expression during early development is unclear. Whole-mount in situ hybridization revealed that k18 was a maternally inherited gene and that its expression is restricted to the single layer of enveloping cells on the surface of embryos during the gastrula stage. At later stages, K18 expression was detected in the epithelial cells, pronephric duct, digestive tract, dorsal aorta, and fins. By using microinjection, we generated the transgenic line $\operatorname{Tg}(\mathrm{k} 18(2.9)$ :RFP), which carries an upstream 2.9-kb segment of $k 18$ gene fused with a red fluorescent protein (RFP) reporter. The spatiotemporal distributions of red fluorescent signal of $\operatorname{Tg}(\mathrm{k} 18(2.9)$ :RFP) line correlated well with endogenous $k 18$ transcripts detected by whole-mount in situ hybridization, indicating that this line is capable of recapitulating endogenous $k 18$ expression patterns. We noticed that the red fluorescence appeared strongly in the dorsal, pectoral, pelvic, anal, and caudal fins when transgenic fish became adults. Interestingly, we also found that when F1 female from the $\operatorname{Tg}(\mathrm{k} 18(2.9)$ :RFP) line were mated with wild-type males, 100\% (326/326) of F2 offspring expressed red fluorescence at the one-cell stage. In contrast, when F1 male from the $\operatorname{Tg}(\mathrm{k} 18(2.9)$ :RFP) line were mated with wild-type females, only 49.8\% (138/277) of F2 embryos exhibited red fluorescence. On the basis of these findings, we suggest that the transcript of zebrafish K18 is inherited as a maternal effect. We believe that $\operatorname{Tg}(\mathrm{k} 18(2.9)$ :RFP) fish should be an excellent experimental animal for studying the zygotic regulatory mechanism of $k 18$.

(C) 2006 Elsevier B.V. All rights reserved.
\end{abstract}

Keywords: Cytokeratin; Epithelial; Maternal effect; Transgenic; Zebrafish

\section{Results and discussion}

Eukaryotic cells (including epithelial cells) contain a complex of different intermediate filaments (IFs): type I and II keratins; type III, vimentin, peripherin and desmin; type IV, $\alpha$-internexin and neurofilaments; type V, lamins; and type VI, nestin (Parry and Steinert, 1992; van de Klundert et al., 1993). Of all the IF proteins, keratin IFs are the most complex and are considered as the hallmark proteins of epithelial cell differentiation (Singh and Gupta, 1994).

Cytokeratin 18 (K18) is an ' $\mathrm{S}$ ' keratin and is the first of the IF proteins to be expressed during mammalian (Moll et al., 1982) and amphibian (Gard and Klymkowsky, 1998) development. In addition to higher vertebrates, the cDNA sequences of $k 18$ are also available from several fish models important to

\footnotetext{
* Corresponding author. Tel.: +88622364 1705; fax: +886223638483.

E-mail address: hjtsai@ntu.edu.tw (H.-J. Tsai).

${ }^{1}$ Equal contribution.
}

1567-133X/\$ - see front matter (C) 2006 Elsevier B.V. All rights reserved. doi:10.1016/j.modgep.2005.10.002 developmental research, such as shark (Schaffeld et al., 1998), carp (Garcia et al., 2005), trout (Markl and Franke, 1988; Schaffeld et al., 2002), goldfish (Druger et al., 1994), and zebrafish (Schaffeld et al., 2003). However, little is known about fish K18 expression during early development, especially during zebrafish embryogenesis. In this article, we report the endogenous zebrafish $k 18$ expression patterns during early development. Additionally, we generated the transgenic zebrafish line $\operatorname{Tg}(\mathrm{k} 18(2.9)$ :RFP), which is able to recapitulate endogenous $k 18$ gene expression.

\subsection{Expression patterns of K18 during early embryonic development}

To determine the spatiotemporal expression patterns of $k 18$ during early development, we performed whole-mount in situ hybridization using a $k 18$ antisense digoxigenin (DIG)-labeled riboprobe. Zebrafish $k 18$ transcripts were first observed at the one-cell stage (Fig. 1A), and expression extended to all the surface cells of the enveloping layer of the embryo during gastrulation (Fig. 1B-E). Zebrafish $k 18$ expression became 


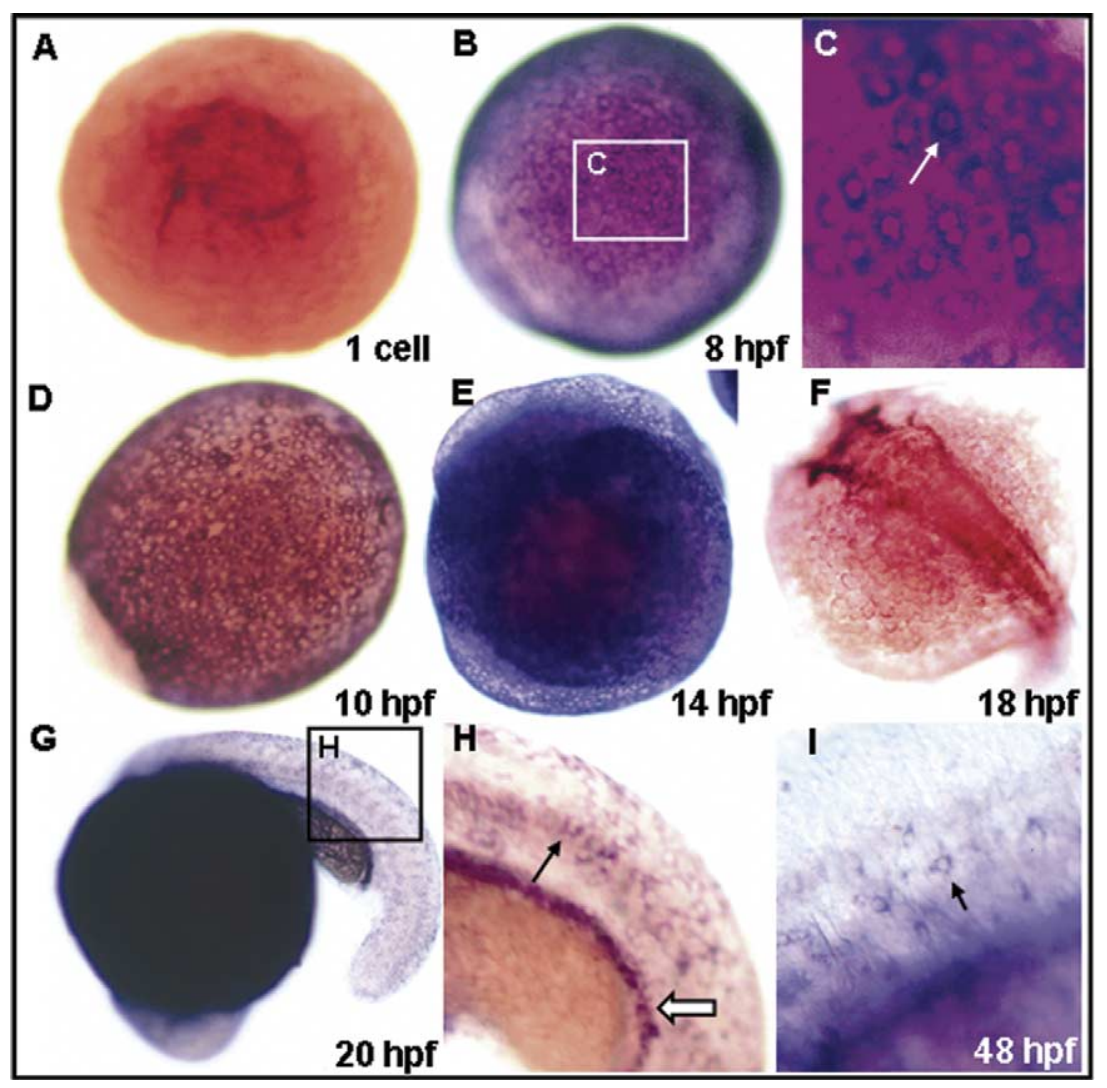

Fig. 1. Cytokeratin 18 (K18) expression during early embryonic stages. (A) At the one-celled stage, dorsal view. (B) At $8 \mathrm{~h}$ postfertilization (hpf), dorsal view. (C) Enlarged view of the same embryo in B. (D) At 10-hpf, dorsal view. (E) At 14-hpf, lateral view. (F) At 18-hpf, dorsal view. (G) At 20-hpf, lateral view. (H) Enlarged view of the same embryo in G; the arrow indicates the presumptive epithelial cells. (I) Detailed view of 48 -hpf embryos; the arrow indicates the presumptive epithelial cells.

restricted in the future head region at the $18 \mathrm{~h}$ postfertilization (hpf; Fig. 1F). Expression of zebrafish $k 18$ continued during 20 to 48-hpf in the epithelial cells and pronephric duct (Fig. 1G-I).

\subsection{K18: RFP transgenes are stably inherited}

We injected zebrafish embryos with the upstream 2.9-kb segment of the $k 18$ gene fused with the RFP reporter gene, selected the RFP-positive F0 embryos, and raised them to adulthood. We identified germ-line transmission by examining embryos after crossing with wild-type and observed the appearance of red fluorescence. One male F0 founder was screened to generate RFP-positive F1 embryos. The F2 inheritance rate for this transgenic line is 50.15\% (168 RFPpositive of 335 total embryos), indicating there was a single insertion site of the transgene in the genome.

\subsection{Zebrafish K18 transcripts are maternally inherited}

All of the F2 offspring (100\%, 326/326) produced by F1 $\operatorname{Tg}(k 18$ (2.9):RFP) females of mated with wild-type males showed red fluorescence from the one-cell stage (Fig. 2A) to segmentation stages (Fig. 2B). Red fluorescence became stronger and extended to the whole body at 3 days postfertilization (dpf; Fig. 2D). At 4 dpf, the red signals gradually diminished to undetectable level (Fig. 2E) in 46\% (108 out of
235) of F2 offspring. Moreover, 54\% (127 out of 235) of them still displayed red fluorescence. In contrast, all of the F2 embryos produced by $\mathrm{F} 1 \mathrm{Tg}(\mathrm{k} 18(2.9)$ :RFP) males mated with wild-type females showed no red fluorescence at the one-cell stage (data not shown), yet $49.8 \%$ (138/277) of them began to display red fluorescence at $14 \mathrm{hpf}$ (Fig. 2C). Reverse transcription-polymerase chain reaction (RT-PCR) demonstrated a high expression level of the endogenous $k 18$ mRNA in F2 embryos from the one-cell stage to $24 \mathrm{hpf}$ (data not shown). However, embryos that received only the paternal transgene exhibited no RFP expression at the one-cell stage (data not shown). $r f p$ mRNA was detected as early as $3 \mathrm{hpf}$ indicating that zygotic $k 18$ transcription was initiated at the mid-blastula transition. Taken together, we propose that the $k 18$ transcripts are maternally inherited.

Several maternally produced zebrafish keratin genes have been identified, such as keratin 4 (K4; formerly was named K8; Imboden et al., 1997; Gong et al., 2002; Schaffeld et al., 2003) and cytokeratin II (CKII; Chua and Lim, 2000). At later developmental stages, a high level of zebrafish k18 transcripts was detected in pronephric duct and dorsal aorta. Comparison with the newly identify type I keratin DAPK-I (Jiang et al., 2003) revealed that both k18 and DAPK-1 were expressed in the dorsal aorta and pronephric duct. However, only k18 mRNA and not DAPK-1, was maternally produced. 


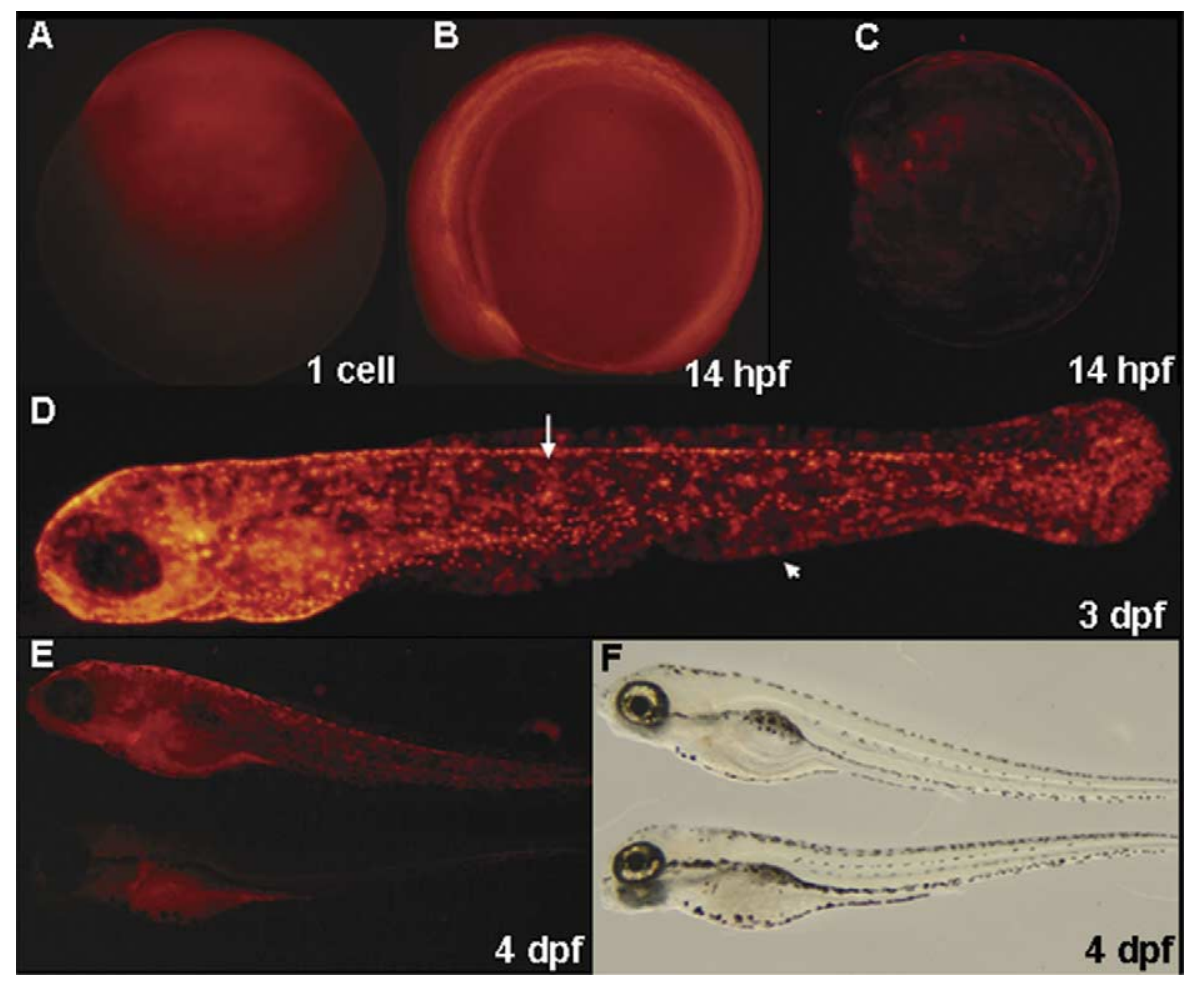

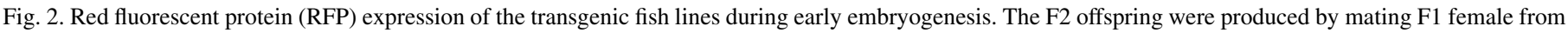

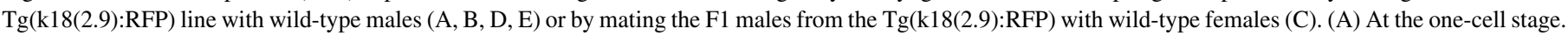

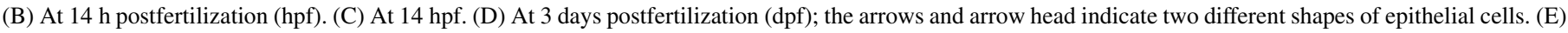

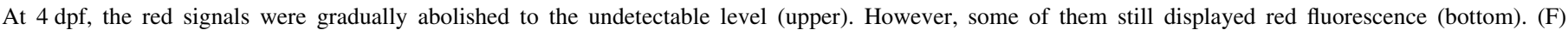
Observation under the light field.

Maternal products are produced during oogenesis and stored in the mature oocyte and can initiate developmental cascades that carry out the embryonic developmental program (Pelegri, 2003). The roles of maternal factors in zebrafish, such as alk8 (Payne-Ferreira et al., 2004), tcf-3 (Kim et al., 2000), smoothened (Varga et al., 2001) and one eyed pinhead (Gritsman et al., 1999) have been reported. Here, for the first time, we demonstrate that an upstream 2.9 -kb segment of the $k 18$ gene is capable of driving $k 18$ expression before mid-blastula transition and is sufficient to recapitulate the endogenous k18 transcription. The mechanism of transcriptional regulation of maternally inherited genes is hard to study because of the interference of maternally produced mRNA. Here, we generated $\operatorname{Tg}(\mathrm{k} 18(2.9): \mathrm{RFP})$ fish and studied the regulatory mechanism. For example, F2 embryos displayed red fluorescence produced by mating $\operatorname{Tg}(\mathrm{k} 18(2.9)$ :RFP) males and wild-type females displayed red fluorescence and can be used as a great tool for studying zygotic expression. This transgenic line should provide new insights into k18 expression at the transcription level in the early embryogenesis.

\subsection{Tissue distribution of transgenic line K18: RFP}

Cryosectioning of $48 \mathrm{hpf}$ embryos from the $\operatorname{Tg}(\mathrm{k} 18(2.9)$ : RFP) line revealed RFP signals distributed in the dorsal aorta, gut, and pronephric duct (Fig. 3A), as well as endogenous k18 expression (Fig. 3B). At 7 dpf, transgenic RFP expression was detected in the developing eye region (Fig. 3C and D), epidermis, dorsal aorta (Fig. 3E), pronephric duct (Fig. 3E), and dorsal fin (Fig. 3E). In adult fish, red fluorescence was observed on the surface of the fish body; on the dorsal, pectoral, pelvic, anal, and caudal fins (Fig. 4A-C); and especially in the epidermis cells of the scales (Fig. 4D). When the scale was removed, no red fluorescence was found in the skin (Fig. 4E). From these observations, we conclude that $\operatorname{Tg}(\mathrm{k} 18(2.9)$ :RFP) fish were able to recapitulate the endogenous $k 18$ expression patterns.

In addition, this $\operatorname{Tg}(\mathrm{k} 18(2.9)$ :RFP) fish line will be an excellent experimental model for studying fin regeneration. After amputation of the zebrafish fin, several genes are upregulated or differentially expressed and are responsible for the complete restoration of the fin structure. Padhi et al. (2004) reported that $k 18$ is up-regulated at 1 day postamputation (dpa), whereas $k 4$ is up-regulated at 4 dpa. Based on these observations, we believe that the $\operatorname{Tg}(\mathrm{k} 18(2.9)$ :RFP) transgenic line should be a good alternative material for studying fin regeneration.

\section{Experimental procedures}

\subsection{Experimental fish}

Zebrafish $\mathrm{AB}$ strains were kept under a 14-h light and 10-h dark photoperiod at approximately $28.5^{\circ} \mathrm{C}$. After fertilization, the eggs were collected and cultured in an aquarium. Embryonic cleavage number and somite 


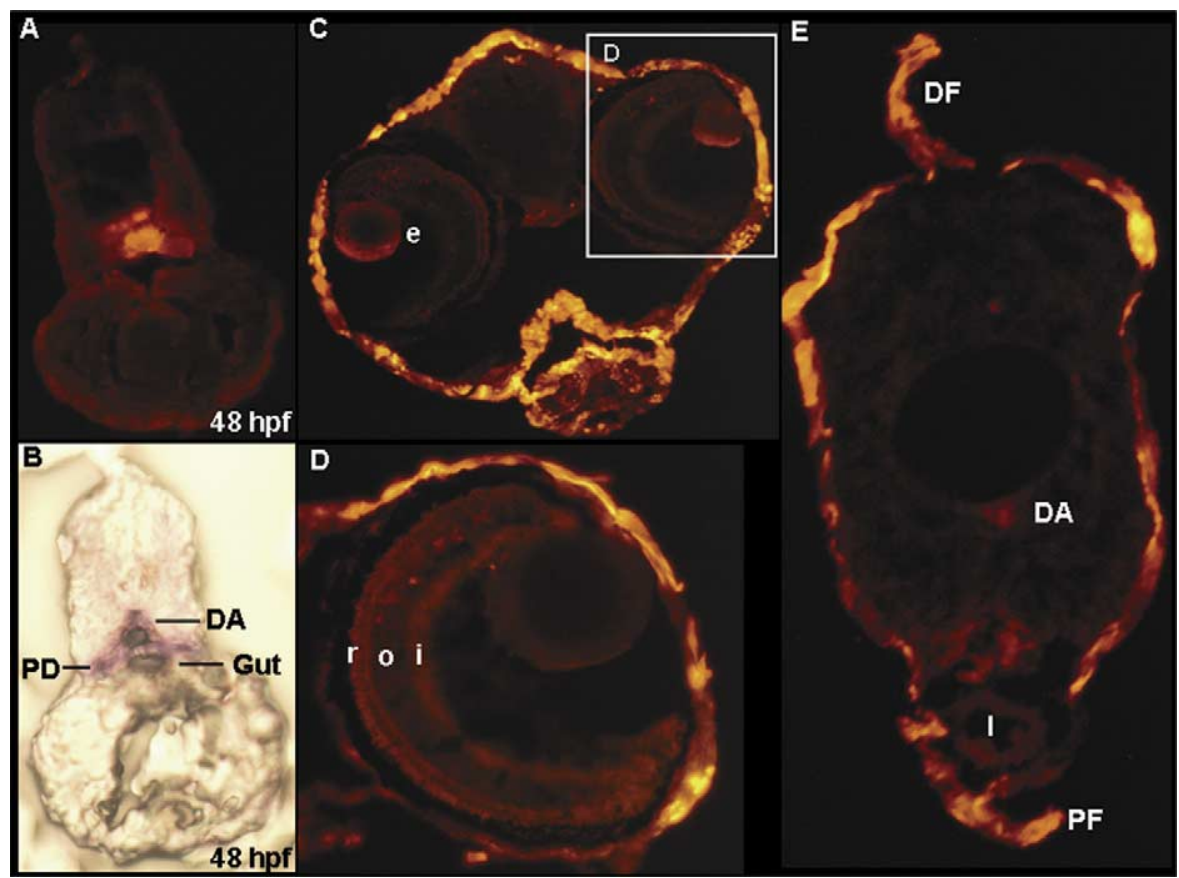

Fig. 3. Cryosections of $48 \mathrm{~h}$ postfertilization (hpf) and 7 days postfertilization (dpf) larva from wild-type embryos and Tg(k18(2.9):RFP) transgenic line embryos. (A) Cryosection of the wild-type $48 \mathrm{hpf}$ embryos, revealing that the endogenous k18 was expressed in the dorsal aorta, pronephric duct, and gut. (B) The comparable section from a 48 hpf k18(2.9):RFP transgenic embryo. (C) Head region. (D) Enlarged view of the same section in C. (D) Trunk section. DA, dorsal aorta; DF, dorsal fin; I, intestine; PD, pronephric duct; PF, pelvic fin; e, eye; i, inner plexiform layer; o, outer plexiform layer; r, rods and cones.

formation were observed with a light microscope to determine the developmental stages (Kimmel et al., 1995).

\subsection{Digital cloning of zebrafish K18 upstream regulatory regions}

We used 'MSLRTSYSVRSSTSQVPVSQVSIKR', which corresponds to zebrafish k18 (Schaffeld et al., 2003) amino acid positions from 1 to 25 , to blast the DNA database on the following Web site (http://www.ensembl.org/Multi/ blastview? ?species = Danio_rerio) with 'tBlastX' as the search tool and 'nearexact match' as the search sensitivity.

\subsection{Genomic DNA isolation and plasmid construction}

DNA was extracted from the caudal fin pools of seven independent adult zebrafish by using standard procedures (Westerfield, 1995). A 3.7-kb zebrafish K18 upstream regulatory region was amplified from zebrafish genomic DNA by using primers k18-3Kf (AGGACATCTGCCCTCCAGCAC) and k18-3Kr (CTCGCTGGTGTAAGTGAGCAGACG). Thirtyfive cycles of PCR amplification were performed by Taq DNA polymerase (Viogene). Each cycle consisted of denaturation for $40 \mathrm{~s}$ at $94{ }^{\circ} \mathrm{C}, 40 \mathrm{~s}$ of annealing at $60^{\circ} \mathrm{C}$, and $3 \mathrm{~min} 30 \mathrm{~s}$ of extension at $72^{\circ} \mathrm{C}$. The last extension step was extended for $15 \mathrm{~min}$ at $72{ }^{\circ} \mathrm{C}$. Amplified DNA fragments were

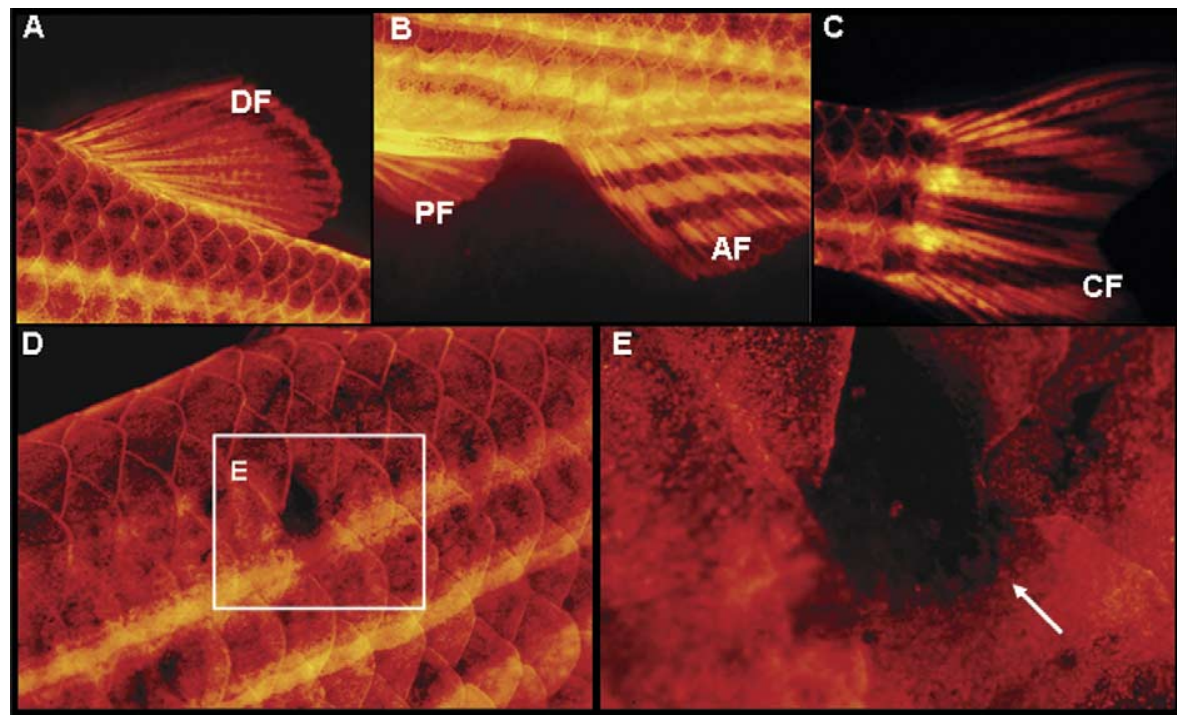

Fig. 4. (A-E) Red fluorescent protein (RFP) expression patterns of transgenic lines in the adult fish. The arrow indicates that no red fluorescence was observed when the scale was removed. AF, anal fin; $\mathrm{CF}$, caudal fin; DF, dorsal fin; $\mathrm{PF}$, pelvic fin. 
ligated to pGEM T-Easy vector (Promega) to produce pT7K18. Then, pT7K18 was digested by StuI (NEB), and a 2.9-kb StuI-digested fragment was purified and ligated to SmaI-digested pDsRed2.1 (Clonetech) vectors to generate pZK18RFP.

\subsection{DNA preparation for microinjection and transient RFP expression}

Plasmid pZK18RFP was digested by XhoI (NEB). The procedures of DNA purification, microinjection and transient red fluorescence detection were each performed as previously described (Chen et al., 2003). Transgenic embryos were observed hourly, especially from 1 to $14 \mathrm{hpf}$, under a stereo dissecting microscope (MZ12, Leica) equipped with a fluorescent module having a DsRed filter cube (Kramer Scientific). Photographs were taken with a S2 Pro digital camera (Fuji) when embryos developed at specific stages.

\subsection{Identification of germ-line transmitted zebrafish}

All RFP-positive embryos at $24 \mathrm{hpf}$ were raised to adulthood. Transgenic founders (F0) were mated with wild-types to confirm the parental individual that was capable of transmitting k18:RFP to the following offspring. At least 200 embryos from each pair were examined for the appearance of red fluorescence. After screening, RFP-positive F1 embryos were raised to adulthood and crossed with wt zebrafish to generate a heterozygotic F2 generation.

\subsection{RNA isolation and RT-PCR}

Embryos were homogenized with TRIzol reagent (Gibco BRL), and their RNAs were extracted according to the manufacturer's instructions (Gibco BRL). First-strand cDNA was synthesized using the SuperScript Preamplification System (Gibco BRL). Primers, K18F (GTCCACGACTACAGCCGCTTCCAGC) and K18R (GTAGGTGGCGATTTCTGCCTCCAGC) were synthesized according to the nucleotide positions 459-483 and 1202-1232 of k18 cDNA (accession no. NM178437), for detecting the endogenous K18 (Schaffeld et al., 2003). Finally, the RT-PCR product of K18 was sub-cloned to pGEMT-easy vector (Promega) and was ready to use for synthesizing k18 antisense DIG-labeled riboprobe.

\subsection{Cryosection and whole-mount in situ hybridization}

The procedures of cryosection and whole-mount in situ hybridization were described by Chen and Tsai (2002), with the exception that embryos at 1-7 dpf were used.

\section{Acknowledgements}

This project was supported by the National Science Council, Republic of China, under grant numbers of NSC 93-2313-B032-003 (YHC) and NSC 91-2811-B-002-029 (HJT).

\section{References}

Chen, Y.H., Tsai, H.J., 2002. Treatment with myf5-morpholino results in somite patterning and brain formation defects in zebrafish. Differentiation $70,447-456$.

Chen, Y.H., Lee, H.C., Liu, C.F., Lin, C.Y., Tsai, H.J., 2003. Novel regulatory sequence $-82 /-62$ functions as a key element to drive the somite-specificity of zebrafish myf-5. Dev. Dyn. 228, 41-50.

Chua, K.L., Lim, T.M., 2000. Type I and type II cytokeratin cDNAs from zebrafish (Danio rerio) and expression patterns during early development. Differentiation 66, 31-41.
Druger, R.K., Glasgow, E., Fuchs, C., Levine, E.M., Matthews, J.P., Park, C.Y., Schechter, N., 1994. Complex expression of keratins in goldfish optic nerve. J. Comp. Neurol. 340, 269-280.

Garcia, D.M., Bauer, H., Dietz, T., Schubert, T., Markl, J., Schaffeld, M., 2005. Identification of keratins and analysis of their expression in carp and goldfish: comparison with the zebrafish and trout keratin catalog. Cell Tissue Res. 30, 1-12.

Gard, D.L., Klymkowsky, M.W., 1998. Intermediate filament organization during oogenesis and early development in the clawed frog, Xenopus laevis. In: Herrmann, H., Harris, J.R. (Eds.), Intermediate Filaments. Plenum Press, New York, pp. 35-70.

Gong, Z., Ju, B., Wang, X., He, J., Wan, H., Sudha, P.M., Yan, T., 2002. Green fluorescent protein expression in germ-line transmitted transgenic zebrafish under a stratified epithelial promoter from keratin 8. Dev. Dyn. 223, 204-215.

Gritsman, K., Zhang, J., Cheng, S., Heckscher, E., Talbot, W.S., Schier, A.F., 1999. The EGF-CFC protein one-eyed pinhead is essential for nodal signaling. Cell 97, 121-132.

Imboden, M., Goblet, C., Korn, H., Vriz, S., 1997. Cytokeratin 8 is a suitable epidermal marker during zebrafish development. CR Acad. Sci. 320, 689-700.

Jiang, W.S., Kim, E.J., Ro, H., Kim, K.E., Huh, T.L., Kim, C.H., Rhee, M., 2003. Expression of a novel type I keratin, DAPK-1 in the dorsal aorta and pronephric duct of the zebrafish embryos. Gene 312, 145-150.

Kim, C.H., Oda, T., Itoh, M., Jiang, D., Artinger, K.B., Chandrasekharappa, S.C., Driever, W., Chitnis, A.B., 2000. Repressor activity of Headless/Tcf3 is essential for vertebrate head formation. Nature 407, 913-916.

Kimmel, C., Ballard, W.W., Kimmel, S.R., Ullmann, B., Schilling, T.F., 1995. Stages of embryonic development in the zebrafish. Dev. Dyn. 203, 253-310.

Markl, J., Franke, W.W., 1988. Localization of cytokeratins in tissues of the rainbow trout: fundamental differences in expression pattern between fish and higher vertebrates. Differentiation 39, 97-122.

Moll, R., Franke, W.W., Schiller, D.L., Geiger, B., Krepler, R., 1982. The catalog of human cytokeratins: patterns of expression in normal epithelia, tumors and culture cells. Cell 31, 11-24.

Padhi, B.K., Joly, L., Tellis, P., Smith, A., Nanjappa, P., Chevrete, M., et al., 2004. Screen for genes differentially expressed during regeneration of the zebrafish caudal fin. Dev. Dyn. 231, 527-541.

Parry, D.A., Steinert, P.M., 1992. Intermediate filament structure. Curr. Opin. Cell Biol. 4, 94-98.

Payne-Ferreira, T.L., Tong, K.W., Yelick, P.C., 2004. Maternal alk8 promoter fragment directs expression in early oocytes. Zebrafish 1, 27-39.

Pelegri, F., 2003. Maternal factors in zebrafish development. Dev. Dyn. 228, 535-554.

Schaffeld, M., Lobbecke, A., Lieb, B., Markl, L., 1998. Tracing keratin evolution: catalog, expression patterns and primary structure of shark (Scyliorhinus stellaris) keratin. Eur. J. Cell. Biol., 69-80.

Schaffeld, M., Hoffling, S., Haberkamp, M., Conrad, M., Markl, J., 2002. Type I keratin cDNAs from the rainbow trout: independent radiation of keratins in fish. Differentiation 70, 282-291.

Schaffeld, M., Knappe, M., Hunzinger, C., Markl, J., 2003. cDNA sequences of the authentic keratins 8 and 18 in zebrafish. Differentiation 71, 73-82.

Singh, S., Gupta, P.D., 1994. Intermediate filaments—-heterogenous expression pattern and modulation: can their role in structure and function of the cell be ascertained? Biol. Cell 82, 1-10.

van de Klundert, F., Raat, J., Blomendal, H., 1993. Intermediate filaments: regulation of gene expression and assembly. Eur. J. Biochem. 214, 351-366.

Varga, Z.M., Amores, A., Lewis, K.E., Yan, Y.L., Postlethwait, J.H., Eisen, J.S., Westerfield, M., 2001. Zebrafish smoothened functions in ventral neural tube specification and axon tract formation. Development 128, 3497-3509.

Westerfield, M., 1995. The Zebrafish Book, third ed. University of Oregon Press, Oregon. 